https://doi.org/10.22319/rmcp.v11i4.5457

Artículo

\title{
Análisis del pedigrí en diez poblaciones mexicanas de ovinos
}

Joel Domínguez-Viveros ${ }^{a^{*}}$

Felipe Alonso Rodríguez-Almeida ${ }^{\text {a }}$

Adán Medellín-Cázares a

Juan Pablo Gutiérrez-García ${ }^{b}$

${ }^{a}$ Universidad Autónoma de Chihuahua. Facultad de Zootecnia y Ecología. Periférico Francisco R. Almada km 1. 31453, Chihuahua, Chih. México.

${ }^{\mathrm{b}}$ Universidad Complutense de Madrid. Facultad de Veterinaria. Madrid. España.

*Autor de correspondencia: joeldguezviveros@yahoo.com.mx; jodominguez@uach.mx

\section{Resumen:}

Se analizó el pedigrí en diez razas de ovinos mediante parámetros genéticos poblacionales. Las poblaciones fueron Blackbelly (BBL; 19,695), Charollais (CHA; 5,033), Dorper (DOR; 42,171), Dorper Blanco (DOB; 4,213), Dorset (DOS; 5,557), Hampshire (HAM; 12,210), Katahdin (KAT; 77,955), Pelibuey (PEL; 42,256), Rambouillet (RAM; 11,951) y Suffolk (SUF; 14,099); nacidos de 1992 a 2018. Los análisis se realizaron con el software Endog. En padres conocidos, los valores oscilaron de $76.4 \%$ (SUF) a $95.3 \%$ (KAT), con promedio general de $86.0 \%$, los animales con padres desconocidos correspondieron a los fundadores. La población consanguínea (como porcentaje de la población total) fluctuó del $12.3 \%$ en DOS al $48.7 \%$ en DOB, con promedio general de $29.7 \%$; la consanguinidad (F) promedio osciló de 3.9 (KAT) a 14.6 (DOB), con promedio general de 8.0. La evolución de F y sus componentes fue: en todas las poblaciones, la proporción de consanguíneos se incrementó $(P<0.05)$; los niveles de relación genética $(\mathrm{RG})$ son estables; y, la $\mathrm{F}$ presentó tendencias negativas $(P<0.05)$. KAT, DOB y BBL con altas tasas de crecimiento en población consanguínea; DOB y DOS, DOB y CHA con los niveles más altos de F y RG, respectivamente. Seis poblaciones presentaron tamaño efectivo $(\mathrm{Ne})$ mayor a 50; el resto 
presentaron Ne menor a 37, lo cual señala el estatus de cuidado para monitorear la evolución de F y sus posibles implicaciones. El intervalo generacional (IG) osciló de 3.0 a 4.15, con promedio general de 3.45 años, los mayores IG fueron para RAM y SUF, BBL y DOR con IG menores.

Palabras clave: Consanguinidad, Tamaño efectivo, Parámetros poblaciones, Intervalo generacional, Tamaño efectivo, Ancestros fundadores.

Recibido: 17/07/2019

Aceptado: 31/03/2020

\section{Introducción}

En México, la ovinocultura se distribuye en todo el territorio nacional bajo un esquema de tipo regional, condicionada por la disponibilidad de recursos naturales y el mercado ${ }^{(1)}$. El Organismo de la Unidad Nacional de Ovinocultores agrupa a los criadores de ovinos especializados y de registro; coordina el registro genealógico de pureza de raza, así como los programas de mejoramiento genético con base en las evaluaciones genéticas ${ }^{(2)}$. Sin embargo, la selección con base en el mejor predictor lineal insesgado (BLUP), producto de las evaluaciones genéticas, favorece la selección de animales emparentados y por consiguiente incrementos en la consanguinidad ${ }^{(3)}$; además, los niveles de consanguinidad y parentesco están implicados en el proceso de evaluaciones genéticas y las predicciones $\operatorname{BLUP}^{(4,5)}$.

Los esquemas de selección conllevan al impacto de un reducido número de reproductores o familias selectas, generan cambios en la estructura de las poblaciones, así como incrementos en los niveles de consanguinidad, reducción de la variabilidad genética y posibles efectos de deriva genética ${ }^{(6,7)}$. La variabilidad genética determina la capacidad de respuesta a la selección y el progreso genético; identificar los factores que la afectan permite evaluar las acciones realizadas, continuar con el esquema de selección o tomar acciones de corrección ${ }^{(8)}$. El análisis del pedigrí, con base en parámetros genéticos poblacionales, describe la dinámica y variabilidad genética de las poblaciones; la estructura genética de una población permite conocer como se ha realizado el flujo de genes, proporciona información de los ancestros fundadores y sus aportaciones a la variabilidad de la población actual ${ }^{(9,10)}$.

Con base en lo anterior, el objetivo del presente estudio fue analizar el pedigrí y la estructura de la población en diez razas de ovinos mediante parámetros genéticos poblacionales, tales como: integridad del pedigrí, número de generaciones, parentesco y consanguinidad, ancestros y fundadores, número efectivo, intervalo generacional, entre otros. Los resultados 
serán de utilidad para desarrollar esquemas de selección, con el objetivo de optimizar la respuesta a la selección limitando la tasa de perdida de variabilidad genética.

\section{Material y métodos}

La información analizada correspondió a la base de datos del registro genealógico de las poblaciones nacionales de ovinos Blackbelly (BBL), Charollais (CHA), Dorper (DOR), Dorper Blanco (DOB), Dorset (DOS), Hampshire (HAM), Katahdin (KAT), Pelibuey (PEL), Rambouillet (RAM) y Suffolk (SUF); los pedigrí fueron de individuos nacidos en el periodo de 1992 a 2018; en el Cuadro 1 se describe la información genealógica utilizada; los análisis de los pedigrís se realizaron con el software Endog $\mathrm{v} 4.0^{(11)}$ y los parámetros genéticos poblacionales evaluados fueron:

Cuadro 1: Estructura del pedigrí, coeficiente de relación promedio (CRP) y niveles de consanguinidad en diez poblaciones de ovinos mexicanos

\begin{tabular}{|c|c|c|c|c|c|c|c|c|}
\hline Ítem & Pedigrí & $\begin{array}{l}\text { Padres } \\
\text { (PS) }\end{array}$ & $\begin{array}{l}\text { Madres } \\
(\text { PM) }\end{array}$ & $\begin{array}{l}\text { SM\% } \\
(\mathrm{M} / \mathrm{S})\end{array}$ & $\begin{array}{l}\text { Fi } \\
(\mathbf{P F i})\end{array}$ & $\begin{array}{l}\text { Fm } \\
(\text { PFm })\end{array}$ & $\begin{array}{l}\beta_{P} \\
\beta_{F}\end{array}$ & CRP \\
\hline BBL & 19,695 & $\begin{array}{l}544 \\
(29.7)\end{array}$ & $\begin{array}{l}5,847 \\
(2.8)\end{array}$ & $\begin{array}{l}32.4 \\
(10.7)\end{array}$ & $\begin{array}{l}26.8 \\
(8.4)\end{array}$ & $\begin{array}{l}16.9 \\
(9.1)\end{array}$ & $\begin{array}{l}5.6 x * \\
-0.29 x\end{array}$ & 0.88 \\
\hline CHA & 5,033 & $\begin{array}{l}266 \\
(17.5)\end{array}$ & $\begin{array}{l}1,433 \\
(3.4)\end{array}$ & $\begin{array}{l}33.8 \\
(5.4)\end{array}$ & $\begin{array}{l}45.8 \\
(8.0)\end{array}$ & $\begin{array}{l}36.5 \\
(10.1)\end{array}$ & $\begin{array}{l}5.6 x * \\
-0.56 x *\end{array}$ & 3.06 \\
\hline DOR & 42,171 & $\begin{array}{l}1,571 \\
(24.1)\end{array}$ & $\begin{array}{l}12,818 \\
(2.9)\end{array}$ & $\begin{array}{l}34.1 \\
(8.2)\end{array}$ & $\begin{array}{l}26.9 \\
(6.1)\end{array}$ & $\begin{array}{l}17.2 \\
(6.9)\end{array}$ & $\begin{array}{l}6.5 \mathrm{x} * \\
-0.77 \mathrm{x} *\end{array}$ & 0.66 \\
\hline DOB & 4,213 & $\begin{array}{l}166 \\
(22.7)\end{array}$ & $\begin{array}{l}1,287 \\
(2.9)\end{array}$ & $\begin{array}{l}34.4 \\
(7.7)\end{array}$ & $\begin{array}{l}48.7 \\
(14.6)\end{array}$ & $\begin{array}{l}32.3 \\
(14.9)\end{array}$ & $\begin{array}{l}5.6 x * \\
-0.59 x *\end{array}$ & 7.78 \\
\hline DOS & 5,557 & $\begin{array}{l}173 \\
(25.5)\end{array}$ & $\begin{array}{l}1,601 \\
(2.8)\end{array}$ & $\begin{array}{l}31.9 \\
(9.3)\end{array}$ & $\begin{array}{l}12.3 \\
(9.8)\end{array}$ & $\begin{array}{l}8.6 \\
(9.9)\end{array}$ & $\begin{array}{l}5.5 x * \\
-0.42 x\end{array}$ & 1.00 \\
\hline HAM & 12,210 & $\begin{array}{l}467 \\
(22.9)\end{array}$ & $\begin{array}{l}3,687 \\
(2.7)\end{array}$ & $\begin{array}{l}33.4 \\
(8.5)\end{array}$ & $\begin{array}{l}21.3 \\
(5.9)\end{array}$ & $\begin{array}{l}12.6 \\
(6.3)\end{array}$ & $\begin{array}{l}4.9 x * \\
-0.54 x *\end{array}$ & 1.13 \\
\hline KAT & 77,955 & $\begin{array}{l}2,927 \\
(27.3)\end{array}$ & $\begin{array}{l}23,844 \\
(3.3)\end{array}$ & $\begin{array}{l}34.3 \\
(8.2)\end{array}$ & $\begin{array}{l}47.8 \\
(3.9)\end{array}$ & $\begin{array}{l}33.5 \\
(4.1)\end{array}$ & $\begin{array}{l}6.5 \mathrm{x} * \\
-0.01 \mathrm{x}^{\mathrm{ns}}\end{array}$ & 1.28 \\
\hline PEL & 42,256 & $\begin{array}{l}1,285 \\
(26.3)\end{array}$ & $\begin{array}{l}13,293 \\
(2.6)\end{array}$ & $\begin{array}{l}34.5 \\
(10.3)\end{array}$ & $\begin{array}{l}22.8 \\
(6.8)\end{array}$ & $\begin{array}{l}13.8 \\
(7.7)\end{array}$ & $\begin{array}{l}7.7 x * \\
-0.24 x *\end{array}$ & 0.47 \\
\hline RAM & 11,951 & $\begin{array}{l}291 \\
(37.1)\end{array}$ & $\begin{array}{l}3,534 \\
(3.1)\end{array}$ & $\begin{array}{l}32.1 \\
(12.1)\end{array}$ & $\begin{array}{l}24.9 \\
(7.4)\end{array}$ & $\begin{array}{l}15.4 \\
(7.5)\end{array}$ & $\begin{array}{l}7.4 \mathrm{x} * \\
-0.17 \mathrm{x} *\end{array}$ & 1.21 \\
\hline SUF & 14,099 & $\begin{array}{l}347 \\
(30.2)\end{array}$ & $\begin{array}{l}4,006 \\
(2.8)\end{array}$ & $\begin{array}{l}30.1 \\
(11.5)\end{array}$ & $\begin{array}{l}19.2 \\
(9.2)\end{array}$ & $\begin{array}{l}14.2 \\
(9.6)\end{array}$ & $\begin{array}{l}1.4 x^{n s} \\
-0.53 x\end{array}$ & 0.86 \\
\hline
\end{tabular}

Pedigrí, total de individuos en el pedigrí. Padres, total de sementales en el pedigrí (PS, promedio de crías por semental). Madres, total de madres en el pedigrí (PM; promedio de crías por madre). Fi, porcentaje de animales consanguíneos (PFi, consanguinidad promedio). Fm, porcentaje de madres consanguíneas (PFm, consanguinidad promedio de las madres).

Pendiente para porcentaje de animales $\left(\beta_{\mathrm{P}}\right)$ consanguíneos y niveles de consanguinidad $\left(\beta_{\mathrm{F}}\right)$;

Poblaciones: Blackbelly (BBL), Charollais (CHA), Dorper (DOR), Dorper Blanco (DOB), Dorset (DOS), Hampshire (HAM), Katahdin (KAT), Pelibuey (PEL), Rambouillet (RAM) y Suffolk (SUF). ns estadísticamente igual a cero $(P>0.05) ; *$ estadísticamente diferente de cero $(P<0.05)$. 


\section{Integridad del pedigrí}

El nivel de integridad se evaluó con base en ${ }^{(8,12)}$ : proporción de ancestros conocidos hasta la tercera generación (padres, abuelos y bisabuelos); número de generaciones completas (NGC), el cual muestra la generación más lejana con dos ancestros conocidos; número de generaciones trazadas (NGT), como indicador del número de generaciones que separan a un individuo de su ancestro más lejano; número de generaciones completas equivalentes (NGE), el cual expresa la suma de todos los ancestros conocidos con base en el número (n) de generaciones que separan al individuo de cada ancestro $\left(\mathrm{NGE}=\Sigma(1 / 2)^{\mathrm{n}}\right)$.

\section{Manejo reproductivo}

PS, promedio de crías por semental; PM, promedio de crías por madres; SM\%, total de sementales y madres, como proporción de la población total del pedigrí; M/S, relación del número de madres / número de sementales.

\section{Consanguinidad (F)}

Con la subrutina mtdfnrm del software $\operatorname{mtdfreml}^{(13)}$ se estimó la consanguinidad de cada individuo (Fi) y de su madre (Fm); con el año de nacimiento de los individuos consanguíneos se generaron las tendencias a través del tiempo, analizando la información de 2010 a 2018. Para el porcentaje de animales consanguíneos $\left(\beta_{\mathrm{P}}\right)$ y consanguinidad promedio $\left(\beta_{\mathrm{F}}\right)$ se realizó un análisis de regresión lineal en el periodo 2010 a 2018, con base en el modelo $\hat{y}=\beta_{0}+\beta x$; donde, $\hat{y}$ correspondió a la variable analizada en el año $x ; \beta_{0}$, el intercepto; $\beta$, corresponde a la pendiente o tasa de cambio; el análisis se realizó con el software para análisis estadístico $\mathrm{SAS}^{(14)}$.

\section{ntervalo generacional (IG)}

Con el planteamiento de la edad media de un animal reproductor, a la que es reemplazado por un descendiente suyo ${ }^{(15)}$; se calculó como la edad promedio de los padres cuando nace su descendencia, a través de las cuatro rutas de selección: padre - hijo, padre - hija, madre hijo y madre - hija ${ }^{(16,17)}$.

\section{Relación genética aditiva promedio (CRP)}

El CRP se obtuvo a partir de la matriz de relaciones genéticas aditivas entre todos los individuos del pedigrí; el cálculo corresponde al valor promedio de los coeficientes de cada individuo con el resto del pedigrí ${ }^{\prime}(9,18)$. 


\section{Número efectivo de fundadores $(f e)$}

Individuo fundador, todo aquel animal con padres desconocidos; el $f e$ se definió como el número de fundadores que, contribuyendo en igual medida, producirían la diversidad genética existente en la población ${ }^{(10,19)}$.

\section{Número efectivo de ancestros $(f a)$}

Ancestro, todo individuo fundador o no, que ha contribuido a la variabilidad genética de la población; el $f a$ se definió como el número de ancestros necesarios para explicar la variabilidad genética total de la población; considera la variabilidad genética aportada por un animal, que no se explica por la contribución de alguno de sus hijos ${ }^{(19,20)}$.

\section{Tamaño efectivo de la población (Ne)}

El Ne realizado se estimó a partir de $1 / 2 \Delta \mathrm{F}$; donde, $\Delta \mathrm{F}$ fue el cambio promedio en consanguinidad y se calculó a partir del número $(\mathrm{t})$ de generaciones completas equivalentes $\left(\Delta \mathrm{F}=1-(1-\mathrm{Fi})^{1 /(\mathrm{t}-1)}\right)$; este Ne considera la cantidad de información genealógica del pedigrí y el traslape generacional ${ }^{(21,22)}$. El Ne define el número de reproductores que podrían generar la consanguinidad calculada y/o tasa de cambio en la varianza genética bajo el esquema de población ideal ${ }^{(10,23)}$.

\section{Resultados y discusión}

La precisión y veracidad en el análisis de la estructura de una población depende de la integridad del pedigrí y el contenido de información genealógica a través de generaciones; con información incompleta, la asignación de individuos a generaciones es aproximada, los cálculos de $\mathrm{F}$ y Ne son imprecisos. En el Cuadro 2 se describe el porcentaje de ancestros, donde por la vía materna se observó mayor contenido de información genealógica. A nivel de padres, los valores oscilaron de $76.4 \%$ (SUF) a $95.3 \%$ (KAT), con un promedio general de $86.0 \%$; los porcentajes de animales con padres desconocidos correspondió al conjunto de animales fundadores. En los pedigrís de las razas Nilagiri, Sandyno ${ }^{(24)}$, Santa Inés ${ }^{(25)}$ y Malpura $^{(17)}$ reportaron integridad y contenido de información genealógica en los niveles del presente estudio; no obstante, para las razas Mehraban ${ }^{(12)}$, Guilan ${ }^{(26)}$ y Morada Nova ${ }^{(8)}$ los pedigrí analizados presentaron porcentajes de padres conocidos por abajo del $60 \%$, para abuelos los niveles fueron menores al $40 \%$ y para bisabuelos las porcentajes fueron menores al $30 \%$; en todos los citados casos, el contenido de información genealógica fue superior por la vía materna. 
Cuadro 2: Porcentaje de progenitores conocidos a través de las diez poblaciones de ovinos mexicanos

\begin{tabular}{lcccccccccc}
\hline & BBL & CHA & DOR & DOB & DOS & HAM & KAT & PEL & RAM & SUF \\
\hline Padres & & & & & & & & & & \\
S & 82.0 & 92.6 & 89.9 & 89.5 & 79.5 & 82.6 & 95.4 & 80.1 & 90.2 & 74.3 \\
M & 83.2 & 95.5 & 90.1 & 89.5 & 80.1 & 81.2 & 95.1 & 80.4 & 91.2 & 78.5 \\
\multicolumn{2}{l}{ Abuelos } & & & & & & & & & \\
SS & 59.6 & 61.6 & 63.4 & 63.9 & 36.4 & 56.9 & 90.7 & 57.4 & 67.3 & 39.1 \\
MS & 58.9 & 68.0 & 64.6 & 63.7 & 42.7 & 58.2 & 89.9 & 57.9 & 70.4 & 42.3 \\
SM & 59.8 & 81.9 & 74.9 & 76.2 & 46.8 & 57.5 & 89.5 & 53.8 & 60.1 & 48.1 \\
MM & 59.2 & 86.4 & 75.2 & 75.3 & 49.4 & 57.6 & 89.1 & 54.3 & 60.6 & 48.1 \\
Bisabuelos & & & & & & & & & \\
SSS & 37.2 & 31.9 & 37.2 & 32.8 & 14.7 & 27.8 & 79.7 & 39.0 & 43.6 & 20.5 \\
MSS & 38.4 & 38.6 & 37.6 & 32.8 & 15.6 & 30.1 & 78.7 & 38.1 & 43.8 & 19.4 \\
SMS & 39.9 & 49.6 & 47.9 & 35.9 & 18.5 & 40.7 & 79.8 & 38.1 & 28.1 & 30.3 \\
MMS & 38.2 & 57.8 & 46.3 & 34.9 & 20.8 & 41.9 & 79.6 & 38.7 & 27.5 & 27.7 \\
SSM & 38.9 & 38.9 & 46.5 & 42.7 & 21.4 & 33.3 & 79.1 & 36.4 & 42.2 & 26.5 \\
MSM & 38.6 & 46.9 & 47.5 & 42.5 & 24.1 & 33.3 & 77.6 & 36.5 & 42.4 & 27.0 \\
SMM & 38.7 & 66.3 & 55.2 & 55.3 & 29.1 & 35.9 & 77.9 & 32.9 & 31.8 & 29.9 \\
MMM & 39.1 & 71.1 & 55.0 & 54.3 & 30.0 & 35.0 & 77.1 & 33.8 & 31.9 & 28.4 \\
\hline
\end{tabular}

Poblaciones: Blackbelly (BBL), Charollais (CHA), Dorper (DOR), Dorper Blanco (DOB), Dorset (DOS), Hampshire (HAM), Katahdin (KAT), Pelibuey (PEL), Rambouillet (RAM) y Suffolk (SUF). Progenitores: S, padre; M, madre.

La integridad del pedigrí se relaciona con las estimaciones de NGC, NGT y NGE (Cuadro 3), donde los valores máximos son similares a través de las diez poblaciones; sin embargo, en los promedios dentro de poblaciones hay marcadas diferencias, los máximos fueron para KAT en contraste con DOS, con los promedios más bajos. La estructura de una población es producto de los esquemas de selección y manejo reproductivo adoptados por los criadores; las diferencias observadas a través de las poblaciones analizadas se pueden atribuir al manejo reproductivo con base en los sementales, con posibles implicaciones en el Ne e IG; los valores de PS y PM (Cuadro 1) muestran la magnitud y la extensión en el uso de los reproductores a través de generaciones, y las estimaciones de SM\% y M/S están relacionadas con la intensidad y presión de selección. 
Cuadro 3: Resultados para el número de generaciones, ancestros fundadores y tamaño efectivo en diez poblaciones de ovinos mexicanas

\begin{tabular}{|c|c|c|c|c|c|c|c|}
\hline Ítem & NGC & NGT & NGE & Anc & Anc\% & Fund & $\mathrm{Ne}$ \\
\hline $\mathrm{BBL}$ & $\begin{array}{l}5.0 \\
(1.66)\end{array}$ & $\begin{array}{l}11.0 \\
(3.23)\end{array}$ & $\begin{array}{l}7.19 \\
(2.24)\end{array}$ & $\begin{array}{l}2,110 \\
(105.0)\end{array}$ & $\begin{array}{l}39 \\
(3.3)\end{array}$ & $\begin{array}{l}3,425 \\
(182.3)\end{array}$ & 36.8 \\
\hline CHA & $\begin{array}{l}4.0 \\
(1.67)\end{array}$ & $\begin{array}{l}11.0 \\
(4.73)\end{array}$ & $\begin{array}{l}5.89 \\
(2.67)\end{array}$ & $\begin{array}{l}235 \\
(35.0)\end{array}$ & $\begin{array}{l}13 \\
(7.1)\end{array}$ & $\begin{array}{l}299 \\
(44.3)\end{array}$ & 22.1 \\
\hline DOR & $\begin{array}{l}5.0 \\
(1.65)\end{array}$ & $\begin{array}{l}12.0 \\
(4.66)\end{array}$ & $\begin{array}{l}6.75 \\
(2.55)\end{array}$ & $\begin{array}{l}2,836 \\
(173.0)\end{array}$ & $\begin{array}{l}74 \\
(3.0)\end{array}$ & $\begin{array}{l}4,219 \\
(226.1)\end{array}$ & 53.8 \\
\hline DOB & $\begin{array}{l}4.0 \\
(1.67)\end{array}$ & $\begin{array}{l}10.0 \\
(3.58)\end{array}$ & $\begin{array}{l}5.61 \\
(2.31)\end{array}$ & $\begin{array}{l}271 \\
(14.0)\end{array}$ & $\begin{array}{l}7 \\
(22.3)\end{array}$ & $\begin{array}{l}441 \\
(16.9)\end{array}$ & 12.2 \\
\hline DOS & $\begin{array}{l}4.0 \\
(1.06)\end{array}$ & $\begin{array}{l}8.0 \\
(2.4)\end{array}$ & $\begin{array}{l}5.0 \\
(1.60)\end{array}$ & $\begin{array}{l}735 \\
(86.0)\end{array}$ & $\begin{array}{l}32 \\
(4.2)\end{array}$ & $\begin{array}{l}1,104 \\
(143.4)\end{array}$ & 50.0 \\
\hline HAM & $\begin{array}{l}4.0 \\
(1.29)\end{array}$ & $\begin{array}{l}10.0 \\
(3.33)\end{array}$ & $\begin{array}{l}5.28 \\
(1.97)\end{array}$ & $\begin{array}{l}1,380 \\
(74.0)\end{array}$ & $\begin{array}{l}28 \\
(4.7)\end{array}$ & $\begin{array}{l}2,090 \\
(124.4)\end{array}$ & 56.8 \\
\hline KAT & $\begin{array}{l}6.0 \\
(2.70)\end{array}$ & $\begin{array}{l}13.0 \\
(6.12)\end{array}$ & $\begin{array}{l}8.03 \\
(4.02)\end{array}$ & $\begin{array}{l}2,578 \\
(109.0)\end{array}$ & $\begin{array}{l}48 \\
(3.9)\end{array}$ & $\begin{array}{l}3,295 \\
(227.6)\end{array}$ & 73.5 \\
\hline PEL & $\begin{array}{l}6.0 \\
(1.50)\end{array}$ & $\begin{array}{l}11.0 \\
(3.12)\end{array}$ & $\begin{array}{l}6.99 \\
(2.10)\end{array}$ & $\begin{array}{l}5,296 \\
(196)\end{array}$ & $\begin{array}{l}94 \\
(3.6)\end{array}$ & $\begin{array}{l}8,348 \\
(349.3)\end{array}$ & 51.5 \\
\hline RAM & $\begin{array}{l}5.0 \\
(1.60)\end{array}$ & $\begin{array}{l}8.0 \\
(2.98)\end{array}$ & $\begin{array}{l}5.78 \\
(2.12)\end{array}$ & $\begin{array}{l}1,073 \\
(93.0)\end{array}$ & $\begin{array}{l}38 \\
(5.6)\end{array}$ & $\begin{array}{l}1,111 \\
(147.7)\end{array}$ & 53.2 \\
\hline SUF & $\begin{array}{l}4.0 \\
(1.09)\end{array}$ & $\begin{array}{l}9.0 \\
(2.60)\end{array}$ & $\begin{array}{l}5.39 \\
(1.65)\end{array}$ & $\begin{array}{l}1,746 \\
(82.0)\end{array}$ & $\begin{array}{l}44 \\
(5.1)\end{array}$ & $\begin{array}{l}3,332 \\
(159.1)\end{array}$ & 34.7 \\
\hline
\end{tabular}

Valores máximos (valores promedio) del número de generaciones completas (NGC), número de generaciones trazadas (NGT) y número de generaciones completas equivalentes (NGE). Anc= total de ancestros (tamaño efectivo de los ancestros). Anc\%= número de ancestros que explican el $50 \%$ de la variabilidad en el pedigrí (máximo porcentaje que un ancestro explica en la variabilidad del pedigrí). Fund= total de fundadores (tamaño efectivo de fundadores). $\mathrm{Ne}=$ tamaño efectivo realizado.

Poblaciones: Blackbelly (BBL), Charollais (CHA), Dorper (DOR), Dorper Blanco (DOB), Dorset (DOS), Hampshire (HAM), Katahdin (KAT), Pelibuey (PEL), Rambouillet (RAM) y Suffolk (SUF).

Con relación a la población consanguínea (Cuadro 1; como porcentaje de la población total) los niveles fluctuaron del $12.3 \%$ en DOS al $48.7 \%$ en DOB, con un promedio general de $29.7 \%$; los niveles de $\mathrm{F}$ (como promedio de la población consanguínea) oscilaron de $3.9 \%$ en KAT a $14.6 \%$ en DOB, con un promedio general de $8.0 \%$. Los niveles y las tendencias de la $\mathrm{F}$, así como sus componentes (CRP, Ne, fe y fa), permiten evaluar la evolución de la variabilidad genética a través del tiempo; los animales consanguíneos son los directamente afectados por los efectos de depresión endogámica y todas las consecuencias que trae consigo el incremento de F. En ovinos, dado la importancia de los efectos maternos ${ }^{(27,28)}$ los posibles efectos de la depresión endogámica también se deben de evaluar a través de los niveles de 
consanguinidad de las madres; en el Cuadro 1, se presenta la proporción de madres consanguíneas y la consanguinidad promedio.

Los escenarios observados en la evolución de F fueron: a) en todas las poblaciones el porcentaje de animales consanguíneos se incrementó a través del tiempo (Cuadro 1; Figura $1)$, con valores $(\beta \mathrm{P})$ en el intervalo de 1.4 a $7.7 \%$; b) sin embargo, los niveles de consanguinidad presentaron tendencias negativas (Cuadro 1; Figura 2), con un valor $\left(\beta_{\mathrm{F}}\right)$ promedio de -0.412 a través de las diez poblaciones; y, c) los niveles del CRP se han mantenido estables (Figura 3). Los esquemas de mejora genética deben de considerar un adecuado equilibrio entre intensidad de selección, consanguinidad y variabilidad genética. Los escenarios en la evolución de la $\mathrm{F}$ se pueden atribuir al uso de reproductores emparentados con gran cantidad de la población y bajos niveles de CRP; la selección con base en BLUP aumenta la probabilidad de selección de animales emparentados; avances en tecnologías reproductivas con lleva a una reducción en el número de progenitores para producir la próxima generación de animales reproductores ${ }^{(3,4,10)}$.

Figura 1: Tendencias de la proporción (\%) de individuos consanguíneos. Blackbelly (BBL), Charollais (CHA), Dorper (DOR), Dorper Blanco (DOB), Dorset (DOS), Hampshire (HAM), Katahdin (KAT), Pelibuey (PEL), Rambouillet (RAM) y Suffolk (SUF)

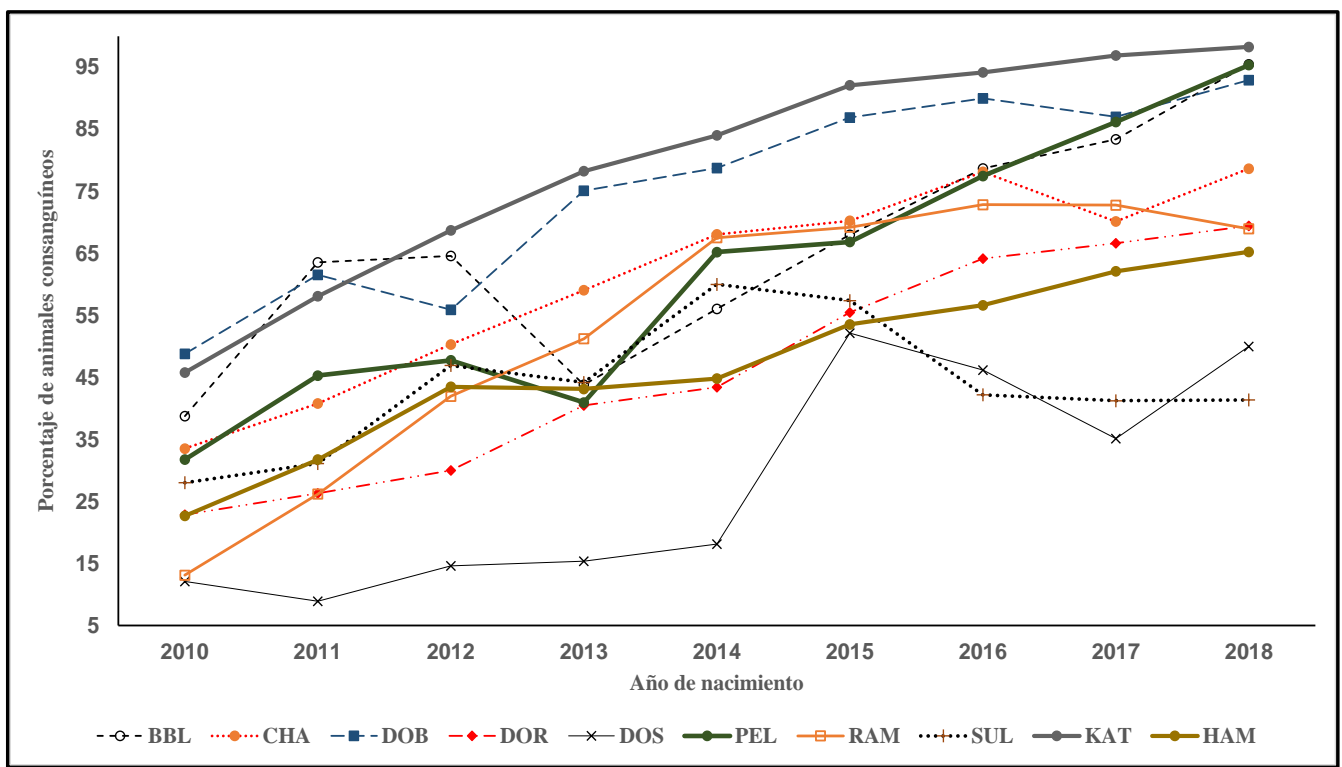


Figura 2: Tendencias de la consanguinidad promedio en la población consanguínea. Blackbelly (BBL), Charollais (CHA), Dorper (DOR), Dorper Blanco (DOB), Dorset (DOS), Hampshire (HAM), Katahdin (KAT), Pelibuey (PEL), Rambouillet (RAM) y Suffolk (SUF). Consanguinidad, niveles de homocigosis en los individuos producto de progenitores emparentados

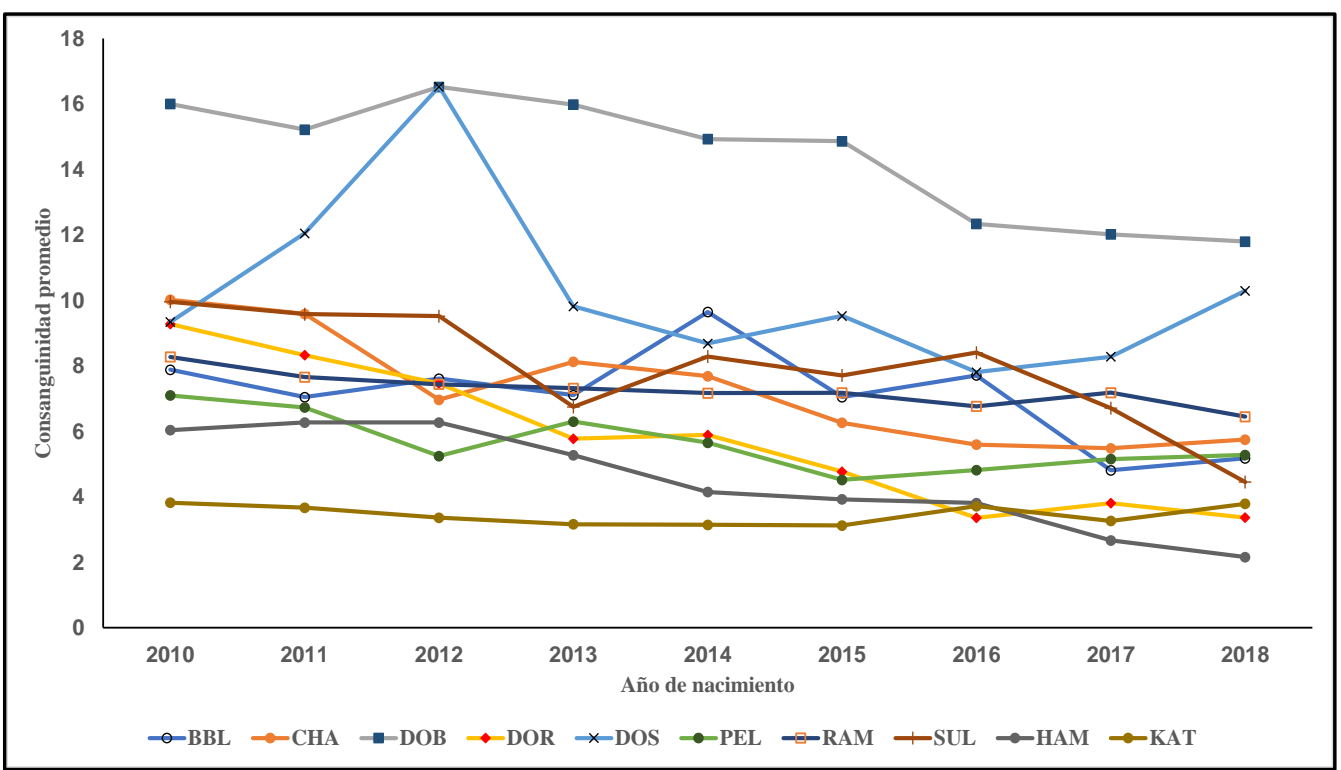

Figura 3: Tendencias del coeficiente de relación promedio. Blackbelly (BBL), Charollais (CHA), Dorper (DOR), Dorper Blanco (DOB), Dorset (DOS), Hampshire (HAM), Katahdin (KAT), Pelibuey (PEL), Rambouillet (RAM) y Suffolk (SUF)

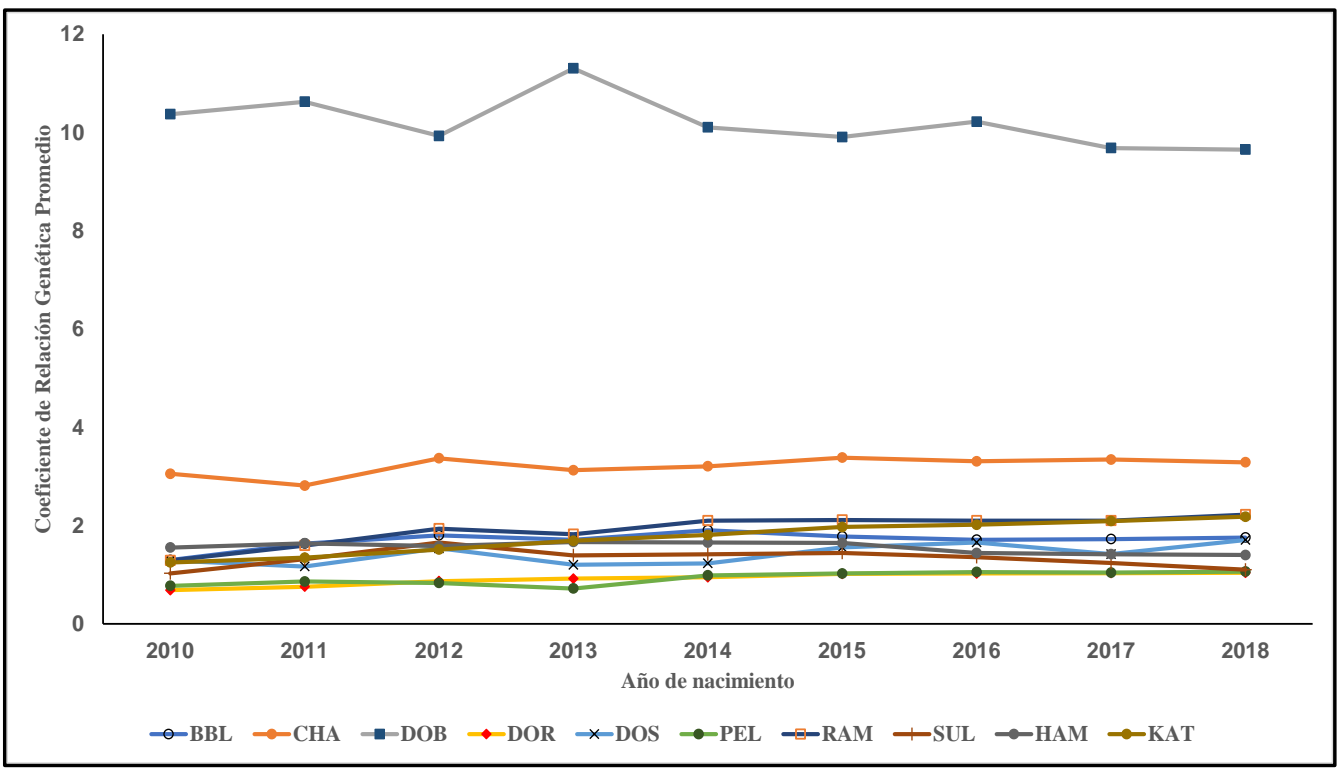


Las poblaciones de KAT, PEL y RAM presentaron las más altas tasas de crecimiento en población consanguínea (Cuadro 1; Figura 1); DOB y DOR presentaron las mayores tendencias negativas de F; y, DOB y CHA tienen los niveles más altos del CRP. En estudios afines con resultados diversos, Danchin-Burge $e a l^{(29)}$ publicaron tendencias de $\mathrm{F}$ cercanas a cero en siete poblaciones de ovinos; sin embargo, Stachowicz et a ${ }^{(10)}$ reportaron tendencias positivas en los niveles de $\mathrm{F}$ en seis razas de ovinos de Canadá; además, Li et al ${ }^{(30)}$, Gowane et $a l^{(17)}$ y Gowane $e t a l^{(18)}$ en ovinos Finnsheep, Merino y Malpura, respectivamente, reportaron tendencias positivas en los niveles de F y CRP.

El concepto de $\mathrm{Ne}$ se desarrolló con base en los lineamientos de la población ideal; el $\mathrm{Ne}$ realizado refleja la acumulación de relaciones genéticas entre individuos y permite predecir los cambios en los niveles de F; además, como resultado de la deriva genética y cambios en las frecuencias génicas presenta los cambios en la varianza genética ${ }^{(31)}$. La estructura de apareamientos y la demografía reproductiva de las poblaciones de ovinos evaluadas difieren a los planteamientos de la población ideal; sin embargo, el Ne realizado tiende a ajustar por algunas de las diferencias ${ }^{(32)}$. Bajos Ne se relacionan con disminución de la variabilidad genética, aumento de apareamientos entre individuos emparentados, fijación de alelos y la mayor reducción en respuesta a la selección ${ }^{(33)}$. El Ne es un concepto básico para el diseño de programas de conservación y/o mejoramiento genético, un Ne menor a 50 es considerado como de cuidado; para el desarrollo de poblaciones recomiendan Ne igual o mayor a 50, el cual considera aumento en los niveles de F menor o igual al $1 \%{ }^{(34)}$; para poblaciones sujetas a mejoramiento genético recomiendan Ne mayores, que permitan optimizar la respuesta a la selección con el mínimo incremento de $\mathrm{F}^{(35)}$. Seis de las poblaciones evaluadas presentaron estimaciones de Ne en el intervalo de 50 a 73.5 (Cuadro 3), lo cual señala que los incrementos de $\mathrm{F}$ serán igual o menores al 1\%; por otro lado, cuatro poblaciones presentaron $\mathrm{Ne}$ en el intervalo de 12.2 a 36.8 , lo cual las coloca en el estatus de cuidado para monitorear la evolución de la F y el CRP, y sus posibles implicaciones en el mejoramiento genético. En otras poblaciones, Leroy et al ${ }^{(32)}$ evaluando los métodos de estimación de $\mathrm{Ne}$ en 40 razas de ovinos, reportaron Ne realizado en el intervalo de 38 a 675, y un valor promedio de 191; asimismo, diversos investigadores ${ }^{(29,30,36.37)}$, que en su conjunto evaluaron el pedigrí de 15 poblaciones de ovinos, reportaron estimaciones de Ne en el intervalo de 55 a 276.

Las relaciones genéticas entre los fundadores y el fe exponen la variabilidad genética inicial; la contribución de los fundadores a la variabilidad del pedigrí exhibe el conjunto de genes que se ha mantenido a través de generaciones ${ }^{(38)}$; en DOB, CHA y SUF, 7, 13 y 44 individuos, respectivamente, explican el 50\% de la variabilidad del pedigrí (Cuadro 3); un reducido número de ancestros que expliquen la variabilidad del pedigrí está asociado a incrementos en los niveles de CRP y F. El fa comprende las posibles causas de pérdida en la variabilidad genética; por lo general $\mathrm{fe}>\mathrm{fa}$, conforme aumenta esa diferencia indica una menor participación de los fundadores a lo largo de generaciones; la relación fe / fa muestra un manejo reproductivo diferencial y considera los posibles cuellos de botella que ha 
experimentado la población. Conforme se incrementa ese cociente indica que la mayoría de los ancestros fueron fundadores, con ausencia de cuellos de botella ${ }^{(39)}$. En el Cuadro 3 se presentan la cantidad de individuos fundadores y ancestros, así como el tamaño efectivo de ambos; la relación fe/fa osciló de 1.2 a 2.0, los cuales coinciden con los resultados publicados por Tahmoorespur y Sheikhloo ${ }^{(16)}$, Ghafouri-Kesbi ${ }^{(40)}$, Mokhtari et $a l^{(36)}$, Mokhtari et $a l^{(37)} \mathrm{y}$ McManus et $a l^{(8)}$ en ovinos Baluchi, Afshari, Kermani, Moghani y Morada Nova, respectivamente.

El CRP puede ser un resumen del manejo reproductivo que ha tenido la población; la F, como consecuencia del apareamiento de animales emparentados, no explica por qué se dieron esos apareamientos; asimismo, el CRP permite diseñar los empadres manteniendo ciertos niveles de $\mathrm{F}$ en la progenie. Las Figuras 2 y 3 muestran la relación entre el CRP y F, al mantenerse los niveles de CRP, no se incrementan los niveles de F; sin embargo, la Figura 1 muestra el manejo reproductivo a través del tiempo, los reproductores provienen de un reducido número de familias y tienden a estar relacionados genéticamente, seleccionados dentro de rebaño con poco flujo a través de rebaños. Por otro lado, en la relación de fe y la F, el CRP de un fundador indica el porcentaje de la población originada por él $\mathbf{l}^{\mathbf{( 1 1 )}}$.

El IG es de suma importancia para validar las pérdidas de variabilidad genética y el progreso genético a través del tiempo; la intensidad de selección, asociada a SM\% y M/S, tiende a reducir el IG, pero resulta en pérdidas de variabilidad genética dado la poca contribución de esos reproductores a la población ${ }^{(9,38)}$. En el Cuadro 4 se presentan las estimaciones del IG por las cuatro vías, los valores oscilaron de 3.0 a 4.15, con un promedio general de 3.45 años y no se observan diferencias sustanciales a través de vías de estimación; sin embargo, a través de poblaciones, las mayores estimaciones fueron para RAM y SUF, en contraste con BBL y DOR con los IG menores. Danchin-Burge et $a l^{(29)}$ en siete razas de ovinos de Francia, reportaron estimaciones de IG promedio de 3.5 años, en un intervalo de 1.9 a 5.0; no obstante, en ovinos $\mathrm{Xalda}^{(19)}$ y en ovinos Somalí ${ }^{(41)}$ reportaron IG promedio de 2.9 y 2.1 años, respectivamente. 
Cuadro 4: Estimaciones del intervalo generacional (años) en diez poblaciones de ovinos mexicanas

\begin{tabular}{lccccc}
\hline Ítem & Padre - hijo & Padre - hija & Madre - hijo & Madre - hija & Media \\
\hline BBL & 3.15 & 3.12 & 3.06 & 3.02 & 3.09 \\
CHA & 3.77 & 3.64 & 3.55 & 3.29 & 3.56 \\
DOR & 3.04 & 3.13 & 3.02 & 3.08 & 3.07 \\
DOB & 3.70 & 3.55 & 3.00 & 3.30 & 3.39 \\
DOS & 3.28 & 3.69 & 3.97 & 3.79 & 3.68 \\
HAM & 3.23 & 3.33 & 3.31 & 3.64 & 3.37 \\
KAT & 3.47 & 3.25 & 3.53 & 3.29 & 3.38 \\
PEL & 3.31 & 3.09 & 3.46 & 3.36 & 3.30 \\
RAM & 3.55 & 4.15 & 3.89 & 4.06 & 3.91 \\
SUF & 3.86 & 3.59 & 3.84 & 3.58 & 3.71 \\
& & & & & \\
Media & 3.44 & 3.45 & 3.46 & 3.44 & \\
\hline
\end{tabular}

Poblaciones: Blackbelly (BBL), Charollais (CHA), Dorper (DOR), Dorper Blanco (DOB), Dorset (DOS), Hampshire (HAM), Katahdin (KAT), Pelibuey (PEL), Rambouillet (RAM) y Suffolk (SUF).

\section{Conclusiones e implicaciones}

Estos resultados muestran un resumen del manejo genético y reproductivo que han realizado los criadores; son de utilidad en el diseño de programas de selección previendo la relación entre respuesta a la selección y los incrementos de consanguinidad, con las consecuencias que conlleva. Los resultados con respecto a las tendencias fueron similares a través de las poblaciones evaluadas: los niveles de consanguinidad tienden a disminuir con pendientes negativas diferentes de cero $(P<0.05)$; las relaciones genéticas fueron estables a través del tiempo; sin embargo, el incremento de la población consanguínea fue significativo, con pendientes positivas diferentes de cero $(P<0.05)$. En magnitud, KAT, PEL y RAM con altas tasas de crecimiento en población consanguínea; DOB y DOS, DOB y CHA con los niveles más altos de consanguinidad y relaciones genéticas, respectivamente; BBL, CHA, DOB, y SUF presentaron bajas estimaciones de tamaño efectivo y se debe monitorear la evolución de la consanguinidad y sus posibles implicaciones.

\section{Literatura citada:}

1. Partida de la PJA, Braña VD, Jiménez SH, Ríos RFG, Buendía RG. Producción de carne de ovina. Instituto Nacional de Investigaciones Forestales, Agrícolas y Pecuarias. Libro técnico No. 5. México. 2013. 
2. Domínguez-Viveros J, Rodríguez-Almeida FA. Resumen de evaluaciones genéticas en ovinos. Catálogo de sementales de alto valor genético de doce razas. Organismo de la unidad nacional de ovinocultores. Universidad Autónoma de Chihuahua. Chihuahua, México. 2017.

3. Verrier E, Colleau J, Foulley JL. Long-term effects of selection based on the animal model BLUP in a finite population. Theo Applied Genet 1993;87:446-454.

4. Wu L, Schaeffer R. Reducing the effect of parent averages from animal solution in mixed model equations. J Anim Breed Genet 2000;117:361-374.

5. Ruiz-Flores A, García-Munguia CA, Núñez-Domínguez R, Ramírez-Valverde R, LópezOrdaz R, García-Muñiz JG. Inclusión del coeficiente de consanguinidad en los modelos de evaluación genética de bovinos Jersey y Suizo Americano en México. Rev Mex Cienc Pecu 2011;2:381-391.

6. Selvaggi M, Dario C, Peretti V, Ciotola F, Carnicella D, Dario M. Inbreeding depression in Leccese sheep. Small Ruminant Res 2010;89:42-46.

7. Vostry L, Milerski M, Schmidova J, Vostra-Vydrova H. Genetic diversity and effects of inbreeding on litter size of the Romanov. Small Ruminant Res 2018;168:25-31.

8. McManus C, Facó O, Shiotsuki L, Jivago de PRJL, Peripolli V. Pedigree analysis of Brazilian Morada Nova hair sheep. Small Ruminant Res 2019;120:37-42.

9. Gutiérrez JP, Altarriba J, Diaz C, Quintanilla R, Cañón J, Piedrafita J. Pedigree analysis of eight Spanish beef cattle breeds. Genet Sel Evol 2003;35:43-64.

10. Stachowicz K, Brito LF, Oliveira HR, Miller SP, Schenkel FS. Assessing genetic diversity of various Canadian sheep breeds through pedigree analysis. Can J Anim Sci 2018;98:741-749.

11. Gutiérrez JP, Goyache F. A note on ENDOG: a computer program for analysis pedigri information. J Anim Breed Genet 2005;122:172-176.

12. Yavarifard R, Hossein-Zadeh NG, Shadparvar AA. Population genetic structure analysis and effect of inbreeding on body weights at different ages in Iranian Mehraban sheep. J Anim Sci Tech 2014;56:31-39.

13. Boldman KG, Kriese LA, Van Vleck DL, Van Tassell CP, Kachman SD. A Manual for use of MTDFREML. A set of programs to obtain estimates of variances and covariances (Draft). USDA. ARS. 1995. 
14. SAS. SAS/STAT User's Guide (Release 9.0). Cary, NC, USA. SAS Inst. Inc. 2005.

15. James JW. A note on selection differentials and generation length when generations overlap. Animal Prod 1977;24:109-112.

16. Tahmoorespur M, Sheikhloo M. Pedigree analysis of the closed nucleus of Iranian Baluchi sheep. Small Ruminant Res 2011;99:1-6.

17. Gowane GR, Ashish C, Misra S, Prince LL. Genetic diversity of a nucleus flock of Malpura sheep through pedigree analyses. Small Ruminant Res 2014;120:35-41.

18. Gowane GR, Prakash V, Ashish C, Prince LL. Population structure and effect of inbreeding on lamb growth in Bharat Merino sheep. Small Ruminant Res 2013;114:7279.

19. Goyache E, Gutiérrez JP, Fernández L, Gómez E, Álvarez I, Diez J, Royo LR. Using pedigree information to monitor genetic variability of endangered populations: the Xalda sheep of Asturias as an example. J Anim Breed Genet 2003;120:95-105.

20. Sheikhlou M, Abbasi MA. Genetic diversity of Iranian Lori-Bakhtiari sheep assessed by pedigree analysis. Small Ruminant Res 2016;141:99-105.

21. Gutiérrez JP, Cervantes I, Molina A, Varela M, Goyache F. Individual increase in inbreeding allows estimating realized effective sizes from pedigrees. Genet Sel Evol 2008;40:359-378.

22. Gutiérrez JP, Cervantes I, Goyache F. Improving the estimation of realized effective population sizes in farm animals. J Anim Breed Genet 2009;126:327-332.

23. Falconer DS, Mackay. TFC Introducción a la genética cuantitativa. Editorial Acribia. Zaragoza, España. 1996.

24. Venkataramanan R, Subramanian A, Sivaselvam SN, Sivakumar T, Sreekumar C, Iyue $M$. Effect of inbreeding and individual increase in inbreeding on growth in Nilagiri and Sandyno breeds of sheep. Animal Genetic Res 2016;58:63-71.

25. Teixeira NMR, Ferreira CJ, Souza CPL, Mendes MCH, Neves FHH. Parâmetros populacionais da raça ovina Santa Inês no Brasil. Pesq Agrop Bras 2013;48:1589-1595.

26. Eteqadi B, Hossein-Zadela NG, Ahad SA. Population structure and inbreeding effects on body weight traits of Guilan sheep in Iran. Small Ruminant Res 2014;119:45-51. 
27. Bradford GE. The role of maternal effects in animal breeding. VII. Maternal effects in sheep. J Anim Sci 1972;35:1324-1334.

28. Gowane GR, Ashish C, Prakash V, Prince LL. The role of maternal effects in sheep breeding: a review. Indian J Small Rumin 2014;20:1-11.

29. Danchin-Burge C, Palhiere I, Francois D, Bibé B, Leroy G, Verrier E. Pedigree analysis of seven small French sheep populations and implications for the management of rare breeds. J Anim Sci 2010;88:505-516.

30. Li MH, Strandén I, Kantanen J. Genetic diversity and pedigree analysis of the Finnsheep breed. J Anim Sci 2009;87:1598-1605.

31. Crow JF, Kimura M. An introduction to population genetic theory. Haper \& Row, New York, USA. 1970.

32. Leroy G, Mary-Huard T, Verrier E, Danvy S, Charvolin E, Danchin-Burge C. Methods to estimate effective population size using pedigree data: examples in dog, sheep, cattle and horse. Genet Sel Evol 2013;45:1-10.

33. Breda FC, Euclydes RF, Silva PC, Robledo de AT, Souza CPL, Rocha SJL, de Almeida TFR, França MAK. Endogamia e Limite de Seleção em Populações Selecionadas Obtidas por Simulação. Rev Brasil Zoot 2004;33:2017-2025.

34. FAO. Secondary guidelines for development of national farm animal genetic resources management plans: management of small populations at risk. Rome, Italy. 1998.

35. Meuwissen THE, Sonesson AK. Maximizing the response of selection with a predefined rate of inbreeding: overlapping generations. J Anim Sci 1998;76:2575-2583.

36. Mokhtari MS, Moradi SM, Esmailizadeh AK, Abdollahi-Arpanahi R, Gutiérrez JP. Genetic diversity in Kermani sheep assessed from pedigree analysis. Small Ruminant Res 2013;114:202-205.

37. Mokhtari MS, Miraei-Ashtiani SR, Jafaroghli M, Gutiérrez JP. Studying genetic diversity in Moghani sheep using pedigree analysis. J Agric Sci Tech 2015;17:1151-1160.

38. Biochard D, Maignel L, Verrier E. The value of using probabilities of gene origin to measure genetic variability in a population. Genet Select Evol 1997;29:5-23.

39. Barros EA, de A Brasil LH, Tejero JP, Delgado-Bermejo JV, Ribeiro MN. Population structure and genetic variability of the Segureña Sheep breed through pedigree analysis and inbreeding effects on growth traits. Small Rumianant Res 2017;149:128-133. 
40. Ghafouri-Kesbi F. Using pedigree information to study genetic diversity and reevaluating a selection program in an experimental flock of Afshari sheep. Arch Tierz 2012;55:375-384.

41. Paiva SR, Olivardo F, Faria DA, Lacerda T, Baretto GB, Carneiro PLS, Lobo RNB, McManus C. Molecular and pedigree analysis applied to conservation of animal genetic resources: the case of Brazilian Somali hair sheep. Trop Animal Health Prod 2011;43:1449-1457. 